\title{
Predicted Janus SnSSe monolayer: a comprehensive first-principle study
}

\author{
San-Dong Guo and Xiao-Shu Guo \\ School of Electronic Engineering, Xi'an University of Posts and Telecommunications, Xi'an 710121, China
}

\begin{abstract}
The Janus structure, by combining properties of different transition metal dichalcogenide (TMD) monolayers in a single polar material, has attracted increasing research interest because of their particular structure and potential application in electronics, optoelectronics and piezoelectronics. In this work, Janus SnSSe monolayer is predicted by means of first-principles calculations, which exhibits dynamic and mechanical stability. By using generalized gradient approximation (GGA) plus spin-orbit coupling (SOC), the Janus SnSSe monolayer is found to be an indirect band-gap semiconductor, whose gap can easily be tuned by strain. High carrier mobilities are obtained for SnSSe monolayer, and the hole mobility is higher than the electron mobility. For SnSSe monolayer, a uniaxial strain in the basal plane can induce both strong in-plane and much weaker out-of-plane piezoelectric polarizations, which reveals the potential as a piezoelectric two-dimensional (2D) material. The high absorption coefficients in the visible light region are observed, suggesting a potential photocatalytic application. Calculated results show that SnSSe monolayer has very high power factor, making it a promising candidate for thermoelectric applications. Our works reveal that the Janus SnSSe structure can be fabricated with unique electronic, optical, piezoelectric and transport properties, and can motivate related experimental works.
\end{abstract}

PACS numbers: 71.20.-b, 77.65.-j, 72.15.Jf, 78.67.-n Keywords: Janus monolayers, Carrier mobility, Piezoelectronics, Power factor

\section{INTRODUCTION}

After the exploration of graphene ${ }^{1}$, the search for new $2 \mathrm{D}$ materials with unique structures and electronic properties have been in progress due to potential applications in the field of optoelectronics and energy conversion and storage. Many 2D materials have been synthesized experimentally or predicted theoretically, such as TMD, groupVA, group IV-VI and group-IV monolayers ${ }^{2-9}$. Among these, TMD monolayers are prominent due to their finite direct band gaps, large on-off ratios, high carrier mobilities and strong optoelectronic responses ${ }^{10,11}$. Due to the lack of inversion symmetry, $2 \mathrm{H}$ TMD monolayers posses a spin-orbit splitting in the valence band, allowing for valley-selective excitation of carriers ${ }^{12}$. Using symmetry analysis, the piezoelectric coefficients of TMD monolayers have been calculated with density functional perturbation theory $(\mathrm{DFPT})^{13}$. Many studies of heat transport properties of TMD monolayers have been reported, and strain effects on their heat transport properties have been investigated $^{14-19}$.

In general, the two-layer chalcogen atoms in TMD monolayers are same element. If the two-layer chalcogen atoms are different, Janus monolayer will be constructed, and then the symmetry will be reduced. Recently, Janus monolayer MoSSe has been successfully synthesised by different experimental strategies, leading to an out-of-plane structural asymmetry ${ }^{5,20}$. Compared to commonly used $2 \mathrm{D}$ materials, the out-of-plane piezoelectric effects of monolayer and multilayer Janus TMD $\mathrm{MXY}(\mathrm{M}=\mathrm{Mo}, \mathrm{W} ; \mathrm{X} / \mathrm{Y}=\mathrm{S}, \mathrm{Se}, \mathrm{Te})$ have been achieved, and out-of-plane piezoelectric coefficient is found to be strongest in multilayer $\mathrm{MoSTe}^{21,22}$. The carrier mobility of monolayer MoSSe can be improved by building bilayer or trilayer structures ${ }^{23}$. The mechanical and elec-

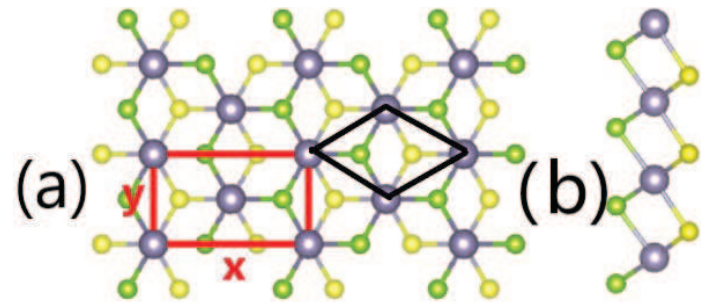

FIG. 1. (Color online) The top view (a) and side view (b) crystal structure of Janus SnSSe monolayer. The large balls represent $\mathrm{Sn}$ atoms, and the small balls for S/Se atoms. The black and red lines represent the rhombus primitive cell and the rectangle supercell.

tronic properties of Janus monolayer MXY $(\mathrm{M}=\mathrm{Ti}, \mathrm{Zr}$, Hf, V, Nb, Ta, Cr, Mo, W; X/Y=S, Se, Te) have been systematically investigated using density functional theory $(\mathrm{DFT})^{24}$. A significant Rashba spin splitting can be induced by intrinsic out-of-plane built-in electric field in monolayer Janus WSeTe ${ }^{25}$. Janus MoSSe and PtSSe monolayers are predicted to be potential wide solarspectrum water-splitting photocatalyst ${ }^{26,27}$. The electronic and optical properties of MoSSe-WSSe vertical and lateral heterostructures have been studied by the firstprinciples calculations ${ }^{28}$. The lattice thermal conductivities of MoSSe/ZrSSe/PtSSe monolayer are predicted by linearized phonon Boltzmann equation, which are much lower than that of the $\mathrm{MoS}_{2} / \mathrm{ZrS}_{2} / \mathrm{PtS}_{2}$ monolayers ${ }^{29-31}$.

In this work, inspiring from the already synthesized $1 \mathrm{~T}$ structure of $\mathrm{SnS}_{2} / \mathrm{SnSe}_{2}{ }^{6,7}$, we systematically investigate the electronic structures, carrier mobilities, piezoelectric properties, optical properties and transport coefficients of Janus SnSSe monolayer. We predict that dynamically 

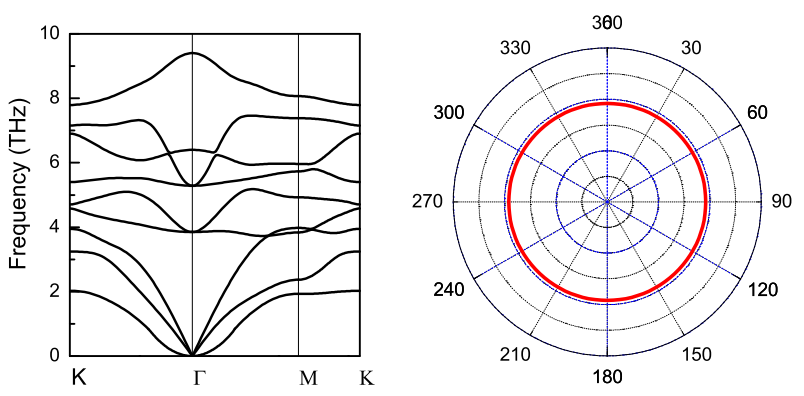

FIG. 2. (Color online) The phonon band dispersion of SnSSe monolayer, and the orientation angle-dependent 2D Young's modulus $C_{2 D}(\theta)$.

TABLE I. For SnSSe monolayer, the lattice constants $a_{0}(\AA)$, the elastic constants $C_{i j}$, shear modulus $G_{2 D}$, Young's modulus $C_{2 D}$ in $\mathrm{Nm}^{-1}$, Poisson's ratio $\nu$ dimensionless and the gaps with GGA and GGA+SOC (eV).

\begin{tabular}{cccc}
\hline \hline$a_{0}$ & $C_{11}=C_{22}$ & $C_{12}$ & $G_{2 D}$ \\
\hline 3.78 & 62.50 & 17.68 & 22.41 \\
\hline \hline$C_{2 D}$ & $\nu$ & Gap & Gap-SOC \\
\hline 57.50 & 0.28 & 0.95 & 0.83 \\
\hline \hline
\end{tabular}

and mechanically stable SnSSe monolayer has distinctive electronic, optical, piezoelectric and transport properties.

\section{COMPUTATIONAL DETAIL}

Within the $\mathrm{DFT}^{32}$, a full-potential linearized augmented-plane-waves method is used to investigate electronic structures of SnSSe monolayer by using WIEN2k code ${ }^{33}$. We use popular GGA of Perdew, Burke and Ernzerhof (GGA-PBE) ${ }^{34}$ as the exchangecorrelation potential, and the SOC was included self-consistently ${ }^{35-38}$. To attain reliable results, we use a $30 \times 30 \times 1 \mathrm{k}$-point meshes in the first Brillouin zone (BZ) for the self-consistent calculation, make harmonic expansion up to $l_{\max }=10$ in each of the atomic spheres, and set $\mathrm{R}_{\mathrm{mt}} * \mathrm{k}_{\max }=8$. The charge convergence threshold is set as $0.0001|e|$ per formula unit, in which $e$ is the electron charge. The electronic transport coefficients of Janus SnSSe monolayer are performed through solving Boltzmann transport equations within the constant scattering time approximation (CSTA), as implemented in the BoltzTrap package ${ }^{39}$. To attain accurate transport coefficients, a $110 \times 110 \times$ $1 \mathrm{k}$-point meshes is used in the first BZ for the energy band calculation. The phonon calculations are carried out by VASP+PHONOPY code ${ }^{40-43}$ with a supercell of $6 \times 6 \times 1$ using the finite displacement method. The kinetic energy cutoff is set to $500 \mathrm{eV}$. We calculate the elastic tensor and piezoelectric stress tensors within $\mathrm{DFPT}^{44}$, using the VASP code.

Due to 3D periodic boundary conditions, the $2 \mathrm{D}$ elastic
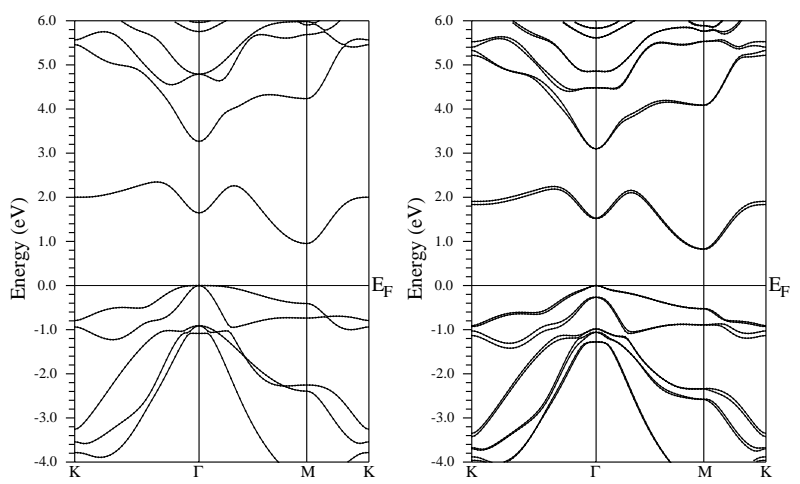

FIG. 3. The energy band structures of SnSSe monolayer using GGA (Left) and GGA+SOC (Right).

coefficients $C_{i j}^{2 D}$ and piezoelectric stress coefficients $e_{i j}^{2 D}$ have be renormalized by the the length of unit cell along z direction $(L z): C_{i j}^{2 D}=L z C_{i j}^{3 D}$ and $e_{i j}^{2 D}=L z e_{i j}^{3 D}$. For $2 \mathrm{D}$ materials, it is noted that the calculated optical properties and electrical conductivity also depend on $L z^{45,46}$, which should be normalized by multiplying $L z / d$, where $d$ for the thickness of $2 \mathrm{D}$ material. However, the $d$ is not well defined like graphene. In this work, the $L z=20 \AA$ is used as $d$.

\section{STRUCTURE AND STABILITY}

The crystal structure of Janus SnSSe monolayer is shown in Figure 1, and the rhombus primitive cell and the rectangle supercell are marked. It is useful for the calculations of carrier mobility and piezoelectric coefficients to use rectangle supercell. With $\mathrm{Pt}$ sandwiched between the $\mathrm{S}$ and Se layers, the symmetry of Janus SnSSe monolayer (No.156) is lower than that of the $\mathrm{SnS}_{2}$ or $\mathrm{SnSe}_{2}$ monolayer (No.164) due to the lack of the reflection symmetry with respect to the central Sn atoms. To avoid spurious interaction between neighboring periodic images, the vacuum space along the $\mathrm{z}$ direction is set to more than $18 \AA$ to separate periodic images. The optimized lattice constants of Janus SnSSe monolayer is $3.784 \AA$ with GGA, being between the ones of $\mathrm{SnS}_{2}(3.69 \AA)$ and $\mathrm{SnSe}_{2}(3.86$ $\AA)^{47}$. To confirm the stability of SnSSe monolayer, the phonon dispersion of Janus monolayer SnSSe is plotted in Figure 2, which shows no imaginary modes, suggesting that monolayer SnSSe is dynamically stable. Due to three atoms in the unit cell, the 3 acoustic and 6 optical phonon branches are observed. Due to $D_{3 h}$ symmetry of SnSSe monolayer, the optical lattice-vibration modes at $\Gamma$ point can be defined as:

$$
\Gamma_{\text {optical }} \equiv \mathrm{A}_{2}^{\prime \prime}(\mathrm{IR})+\mathrm{A}_{1}^{\prime}(\mathrm{R})+\mathrm{E}^{\prime}(\mathrm{IR}+\mathrm{R})+\mathrm{E}^{\prime \prime}(\mathrm{R})
$$

where IR and R mean infrared- and Raman-active mode, respectively. The optical phonon frequencies of SnSSe at $\Gamma$ point are $9.40 \mathrm{THz}\left(\mathrm{A}_{2}^{\prime \prime}\right), 6.40 \mathrm{THz}\left(\mathrm{A}_{1}^{\prime}\right), 5.29$ 
$\mathrm{THz}\left(\mathrm{E}^{\prime}\right)$ and $3.85 \mathrm{THz}\left(\mathrm{E}^{\prime \prime}\right)$. The mechanical stability of SnSSe monolayer can be examined by elastic constants $C_{i j}$. Due to hexagonal symmetry, two independent elastic constants $C_{11}$ and $C_{12}$ are calculated, the $C_{11}=C_{22}=62.50 \mathrm{Nm}^{-1}$ and $C_{12}=17.68 \mathrm{Nm}^{-1}$, and the $C_{66}=\left(C_{11}-C_{12}\right) / 2=22.41 \mathrm{Nm}^{-1}$, which satisfy the Born criteria of mechanical stability ${ }^{48}$, namely

$$
C_{11}>0, \quad C_{66}>0
$$

The Young's modulus $C_{2 D}(\theta)$ can be calculated on the basis of the elastic constants. The Young's modulus $C_{2 D}(\theta)$ along the in-plane $\theta$ can be expressed as follows ${ }^{49}$ :

$$
C_{2 D}(\theta)=\frac{C_{11} C_{22}-C_{12}^{2}}{C_{11} \sin ^{4} \theta+A \sin ^{2} \theta \cos ^{2} \theta+C_{22} \cos ^{4} \theta}
$$

where $A=\left(C_{11} C_{22}-C_{12}^{2}\right) / C_{66}-2 C_{12}$. The $C_{2 D}(\theta)$ is shown in Figure 2, which shows strong mechanical isotropy for SnSSe monolayer. The calculated $C_{2 D}(\theta)$ is $57.50 \mathrm{Nm}^{-1}$, which is smaller than one of MoSSe (113.0 $\left.\mathrm{Nm}^{-1}\right)^{29}$, indicating that monolayer SnSSe is less rigid than MoSSe monolayer. The Poisson's ratio $\nu(\theta)$ is also isotropic, and the calculated $\nu$ is 0.28 . The shear modulus $G_{2 D}$ equals to $C_{66}$. The related data are summarized in Table I. The phonon calculations and elastic constants prove that Janus monolayer SnSSe is dynamically and mechanically stable. Monolayer $\operatorname{SnX}_{2}(\mathrm{X}=\mathrm{S}, \mathrm{Se})$ have been synthesized ${ }^{6,7}$, and MoSSe monolayer have also been successfully fabricated ${ }^{5,20}$. A similar growth method to that of monolayer MoSSe can be used to realize Janus monolayer SnSSe.

\section{ELECTRONIC STRUCTURE}

It has been proved that the SOC has important effects on electronic structures of Janus TMD monolayers ${ }^{50}$. Thus, the SOC is included for electronic structure calculations of Janus SnSSe monolayer, and the related energy bands with GGA and GGA+SOC are plotted in Figure 3. By using GGA, an indirect gap of $0.95 \mathrm{eV}$ with valence band maximum (VBM) at the $\Gamma$ point and conduction band minimum (CBM) at the $\mathrm{M}$ point. It is noted that the bands around VBM are very flat, which can produce very large effective masses of VBM. When including SOC, the gap reduces to $0.83 \mathrm{eV}$, and a spinorbit splitting at the $\Gamma$ point in the valence bands near the Fermi level is $0.266 \mathrm{eV}$.

Strain can effectively tune the electronic structures of Janus TMD monolayers ${ }^{50}$. Here, the effects of biaxial strain on the electronic structures of SnSSe monolayer are examined. To simulate biaxial strain, $a / a_{0}$ is used with $a / a_{0}<1\left(a / a_{0}>1\right)$ being compressive (tensile) strain, where $a$ and $a_{0}$ are the strained and unstrained lattice constant, respectively. The energy band gap (Gap) and spin-orbit splitting value at $\Gamma$ point $(\Delta)$ as a function of $a / a_{0}$ are shown in Figure 4 , and the related energy band structures with $a / a_{0}$ from 0.94 to 1.06 are plotted

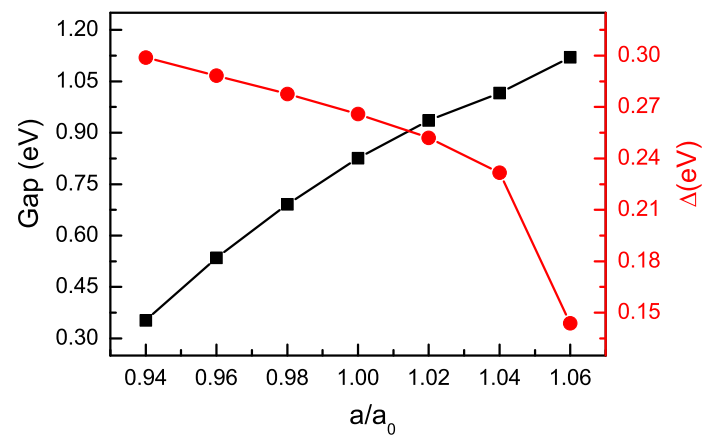

FIG. 4. (Color online) The energy band gap (Gap) and spinorbit splitting value $(\Delta)$ at high symmetry $\Gamma$ point as a function of $a / a_{0}$ by using GGA+SOC.

in Figure 5. It is found that the energy band gap increases monotonously with $a / a_{0}$ changing from 0.94 to 1.06 , which is different from one of other Janus TMD monolayers (up-and-down trend) ${ }^{50}$. With $a / a_{0}$ from 0.94 to 1.04 , the $\Delta$ decrease monotonously, and then a rapid decrease from 1.04 to 1.06 . It is because that the VBM changes from $\Gamma$ point to another point along $\Gamma$-K direction. The strain can tune the positions of conduction band extrema (CBE), and the second $\mathrm{CBE}$ changes from $\mathrm{K}$ point to $\Gamma$ point, when strain varies from compressive strain to tensile one. The conduction bands convergence can be realized by tensile strain, which can be observed at $a / a_{0}=1.06$ point. The more localized valence bands can be induced by tensile strain. These can produce important influence on electronic transport properties of SnSSe monolayer.

\section{CARRIER MOBILITY}

As is well known, the carrier mobility of a semiconductors is an important factor for great potential in the electronics and optoelectronics. To evaluate the carrier mobility, the deformation potential (DP) theory has been proposed by Bardeen and Shockley ${ }^{51}$, which can be used to calculate the intrinsic carrier mobility of $2 \mathrm{D}$ materials. In this theory, the carrier mobility of a $2 \mathrm{D}$ material $\left(\mu_{2 D}\right)$ is defined as:

$$
\mu_{2 D}=\frac{e \hbar^{3} C_{2 D}}{K_{B} T m^{*} m_{d} E_{l}^{2}}
$$

where $T$ is temperature, and $m^{*}$ is the effective mass in the transport direction, and $m_{d}=\sqrt{m_{x} m_{y}}$ is the average effective mass. The $C_{2 D}$ is the elastic modulus derived from $C_{2 D}=\left[\partial^{2} E / \partial^{2} \delta\right] / S_{0}$, where $E$ is the total energy after applying uniaxial strain $\left(\delta=\Delta l / l_{0}\right)$ and $S_{0}$ is the area at equilibrium. In addition, $E_{l}$ is the DP constant defined by $E_{l}=\Delta E / \delta$, where $\Delta E$ is the energy shift of the band edge of CBM or VBM with respect to the vacuum level. After attaining $\mu_{2 D}$, the relaxation time $\tau$ 

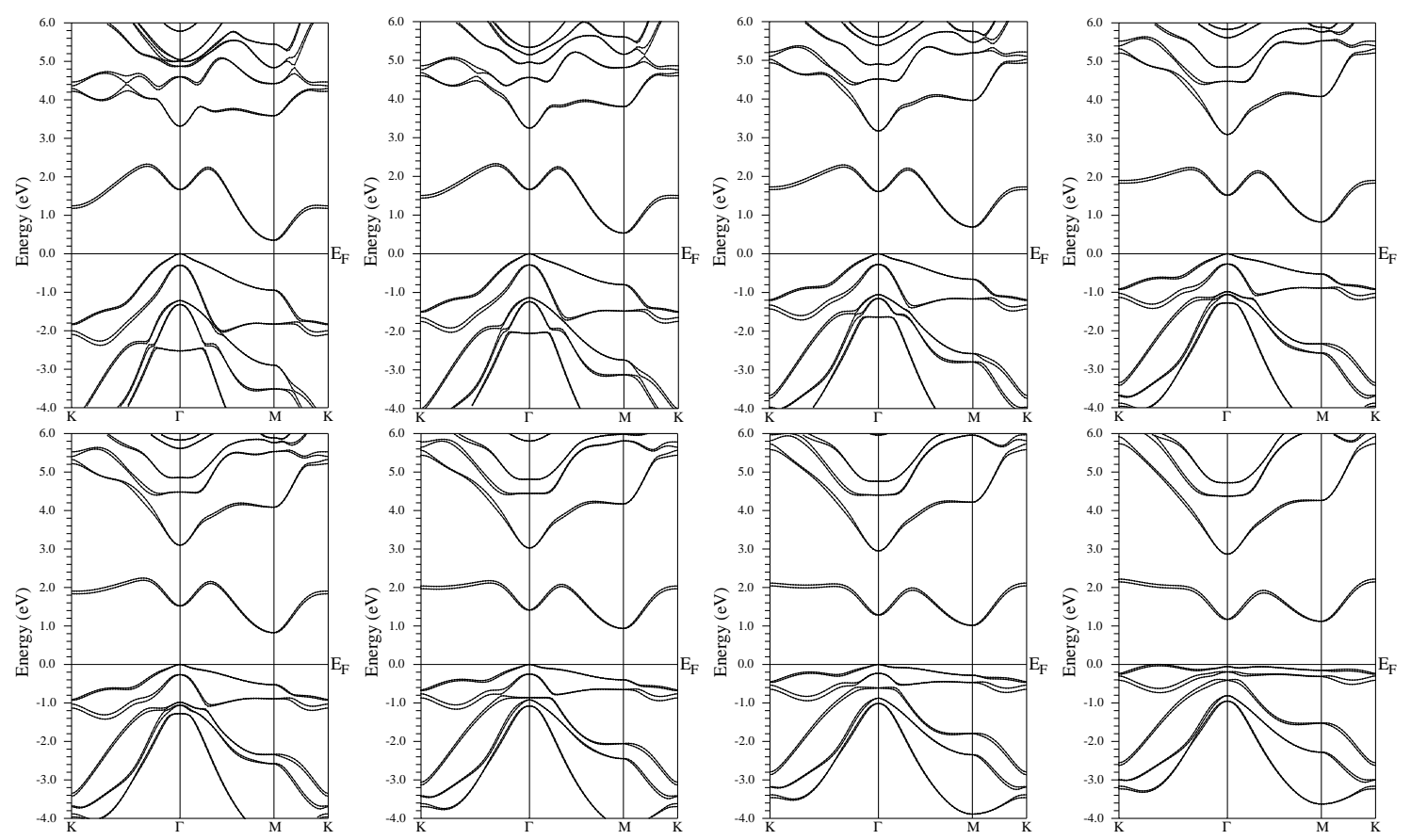

FIG. 5. With $a / a_{0}$ changing from 0.94 to 1.00 (Top) and 1.00 to 1.06 (Bottom), the energy band structures of SnSSe monolayer using GGA+SOC, and the interval is 0.02 .

can be calculated by:

$$
\tau=\mu_{2 D} m^{*} / e
$$

By using DP theory, the carrier mobilities of SnSSe monolayer in both zigzag and armchair directions are calculated, and the rectangular supercell is used with armchair and zigzag being defined as $\mathrm{x}$ and $\mathrm{y}$ directions in Figure 1. The calculated effective masses for electrons and holes with GGA and GGA+SOC are shown in Table II. It is found that the effective masses for electrons between GGA and GGA+SOC are very close, but the ones for holes are very different. These can be explained from their energy structures in Figure 3. When including SOC, the bands near CBM has little change with respect to GGA results, but the flat bands around VBM disappear. Thus, it is very important for calculating effective masses of holes to consider SOC. The DP constant $E_{l}$ is calculated by linearly fitting the band energies of the VBM and CBM with respect to the vacuum energy as a function of $\Delta x / x$ and $\Delta y / y$, which is plotted in Figure 6. On the basis of the calculated effective mass, elastic constant, and deformation potential constant, the carrier mobility and relaxation time for the electrons and holes of monolayer SnSSe along $\mathrm{x}$ and $\mathrm{y}$ directons are calculated, which are shown Table II. Predicted carrier mobilities between electrons and holes show very strong anisotropy, and the weakly anisotropic ones along $\mathrm{x}$ and $\mathrm{y}$ directions for the same carrier type are observed. The hole carrier mobilities of SnSSe monolayer are higher than those of monolayer $\mathrm{SnS}_{2}$ (187.44 $\left.\mathrm{cm}^{2} \mathrm{~V}^{-1} \mathrm{~s}^{-1}\right)$ and $\mathrm{SnSe}_{2}\left(115.65 \mathrm{~cm}^{2} \mathrm{~V}^{-1} \mathrm{~s}^{-1}\right)^{47}$, but its electron carrier mobilities are lower than their ones. The calculated $\tau$ is useful for attaining the electronic transport coefficients of SnSSe monolayer.

\section{PIEZOELECTRIC PROPERTIES}

In noncentrosymmetric materials, the piezoelectric effect can be produced, when mechanical stress is applied. In 2D materials, the third-rank piezoelectric stress tensors $e_{i j k}$ and strain tensor $d_{i j k}$, employing Voigt notation, can be connected by elastic tensor ${ }^{13}$ :

$$
\begin{gathered}
e=d C \\
e=\left(\begin{array}{lll}
e_{11} & e_{12} & e_{16} \\
e_{21} & e_{22} & e_{26} \\
e_{31} & e_{32} & e_{36}
\end{array}\right) \\
d=\left(\begin{array}{lll}
d_{11} & d_{12} & d_{16} \\
d_{21} & d_{22} & d_{26} \\
d_{31} & d_{32} & d_{36}
\end{array}\right) \\
C=\left(\begin{array}{lll}
C_{11} & C_{12} & C_{16} \\
C_{21} & C_{22} & C_{26} \\
C_{61} & C_{62} & C_{66}
\end{array}\right)
\end{gathered}
$$


TABLE II. For SnSSe monolayer, elastic modulus $\left(C_{2 D}\right)$, effective mass $\left(m^{*}\right)$ using GGA+SOC with GGA results given in parentheses, deformation potential $\left(E_{l}\right)$, carrier mobility $\left(\mu_{2 D}\right)$ and relaxation time $(\tau)$ at $300 \mathrm{~K}$.

\begin{tabular}{ccccccc}
\hline \hline Carrier type & & $C_{2 D}\left(\mathrm{Nm}^{-1}\right)$ & $m^{*}$ & $E_{l}(\mathrm{eV})$ & $\mu_{2 D}\left(\mathrm{~cm}^{2} \mathrm{~V}^{-1} \mathrm{~s}^{-1}\right)$ & $\tau(\mathrm{s})$ \\
\hline \hline Electrons & $\mathrm{x}$ & 57.50 & $0.74(0.73)$ & 3.94 & 258.63 & $1.08 \times 10^{-13}$ \\
& $\mathrm{y}$ & 57.50 & $0.23(0.24)$ & -7.49 & 224.71 & $2.99 \times 10^{-14}$ \\
\multirow{2}{*}{ Holes } & $\mathrm{x}$ & 57.50 & $-0.36(-31.44)$ & -4.01 & 580.20 & $1.19 \times 10^{-13}$ \\
& $\mathrm{y}$ & 57.50 & $-0.37(-12.79)$ & -4.10 & 548.74 & $1.14 \times 10^{-13}$ \\
\hline \hline
\end{tabular}
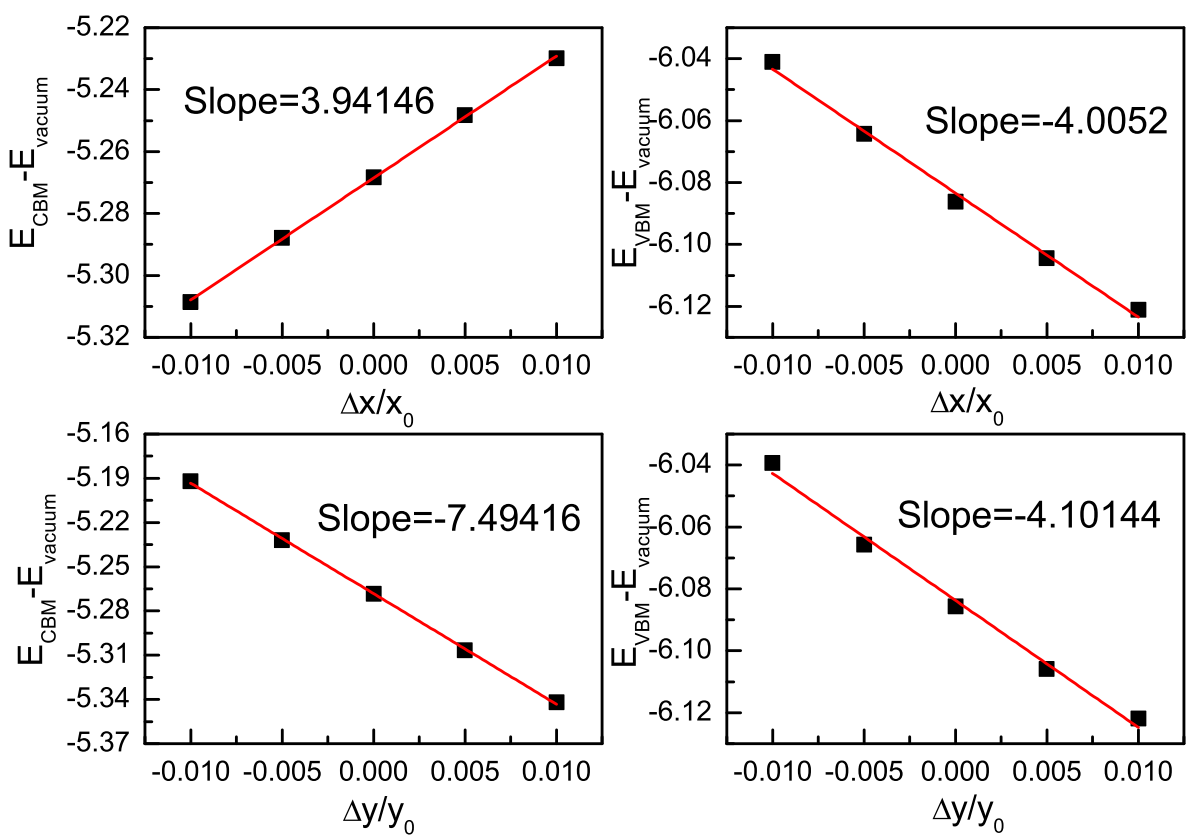

FIG. 6. (Color online)Using GGA+SOC, the band energies of the VBM and CBM of SnSSe monolayer with respect to the vacuum energy as a function of lattice dilation along both $\mathrm{x}$ and $\mathrm{y}$ directions. The red solid lines are linear fitting curves. The fitted slopes are shown, which corresponds to the DP.

Performing symmetry analysis, the number of independent tensor coefficients can be further reduced. Due to a $3 m$ point-group symmetry of SnSSe monolayer, the piezoelectric stress and strain tensors, and elastic tensor become:

$$
\begin{gathered}
e=\left(\begin{array}{ccc}
e_{11} & -e_{11} & 0 \\
0 & 0 & -e_{11} \\
e_{31} & e_{31} & 0
\end{array}\right) \\
d=\left(\begin{array}{ccc}
d_{11} & -d_{11} & 0 \\
0 & 0 & -2 d_{11} \\
d_{31} & d_{31} & 0
\end{array}\right) \\
C=\left(\begin{array}{cccc}
C_{11} & C_{12} & 0 \\
C_{12} & C_{11} & 0 \\
0 & 0 & \left(C_{11}-C_{12}\right) / 2
\end{array}\right)
\end{gathered}
$$

TABLE III. Piezoelectric coefficients $e_{11}\left(d_{11}\right)$ and $e_{31}\left(d_{31}\right)$ of SnSSe and MoSSe Monolayers, and the unit is $10^{-10} \mathrm{C} / \mathrm{m}$ $(\mathrm{pm} / \mathrm{V})$.

\begin{tabular}{lcccc}
\hline \hline MXY & $e_{11}$ & $d_{11}$ & $e_{31}$ & $d_{31}$ \\
\hline \hline SnSSe & 1.009 & 2.251 & -0.091 & -0.114 \\
\hline MoSSe & 3.890 & 4.238 & 0.418 & 0.285 \\
\hline \hline
\end{tabular}

Here, $d_{11}$ and $d_{31}$ are derived by Equation 6, Equation 10, Equation 11 and Equation 12:

$$
d_{11}=\frac{e_{11}}{C_{11}-C_{12}} \quad \text { and } \quad d_{31}=\frac{e_{31}}{C_{11}+C_{12}}
$$

The nonprimitive orthorhombic unit cells of SnSSe monolayer (in Figure 1) is used as the computational unit cell for $e_{i j}$. The calculated $e_{i j}$ and $d_{i j}$ of SnSSe monolayer are shown in Table III, along with ones of already synthesized MoSSe monolayer. Calculated results show that the piezoelectric coefficients of SnSSe are lower than ones of MoSSe. However, the $d_{11}$ is comparable with 


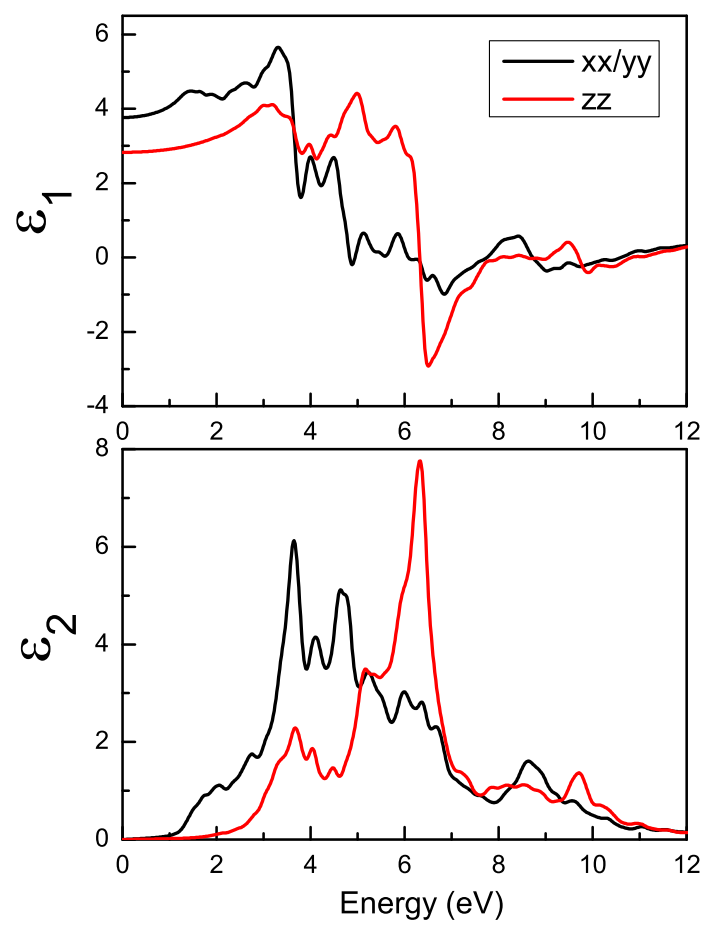

FIG. 7. (Color online)Using GGA+SOC, calculated optical dielectric function of monolayer SnSSe, including real parts (Top) and imaginary parts (Bottom) along xx/yy and $\mathrm{zz}$ directions.

one of $\alpha$-quartz $\left(d_{11}=2.27 \mathrm{pm} / \mathrm{V}\right)^{52}$ commonly used 3D piezoelectric material. It is noted that $\mathrm{SnS}_{2}$ and $\mathrm{SnSe}_{2}$ monolayers display inversion symmetry and therefore no piezoelectricity.

\section{OPTICAL PROPERTIES}

The optical properties of the SnSSe monolayer can be described by the complex dielectric function $\varepsilon(\omega)$, the imaginary part $\varepsilon_{2}(\omega)$ of which is determined by a summation over empty band states as follows ${ }^{53}$ :

$$
\varepsilon_{2}(\omega)=\frac{2 \pi e^{2}}{\Omega \epsilon_{0}} \sum_{k, v, c} \delta\left(E_{k}^{c}-E_{k}^{v}-\hbar \omega\right)\left|<\psi_{k}^{c}\right| u . r\left|\psi_{k}^{v}>\right|^{2}
$$

Where $\epsilon_{0}, \Omega$ and $\hbar \omega$ are the vacuum dielectric constant, the volume and the energy of the incident phonon; The $v$, $c$ and $u$ represents the valence bands, conduction bands and the polarization vector in the incident electric field; The $u . r$ and $\psi_{k}$ is the momentum operator and the wave function at the $\mathrm{k}$ point. The real part $\varepsilon_{1}(\omega)$ of dielectric tensor can be obtained by the Kramers-Kronig relation. According to the dielectric function, the absorption coefficient $\alpha(\omega)$ can be given using the equation ${ }^{54}$ :

$$
\alpha(\omega)=\frac{\sqrt{2} \omega}{c}\left\{\left[\varepsilon_{1}^{2}(\omega)+\varepsilon_{2}^{2}(\omega)\right]^{1 / 2}-\varepsilon_{1}(\omega)\right\}^{\frac{1}{2}}
$$
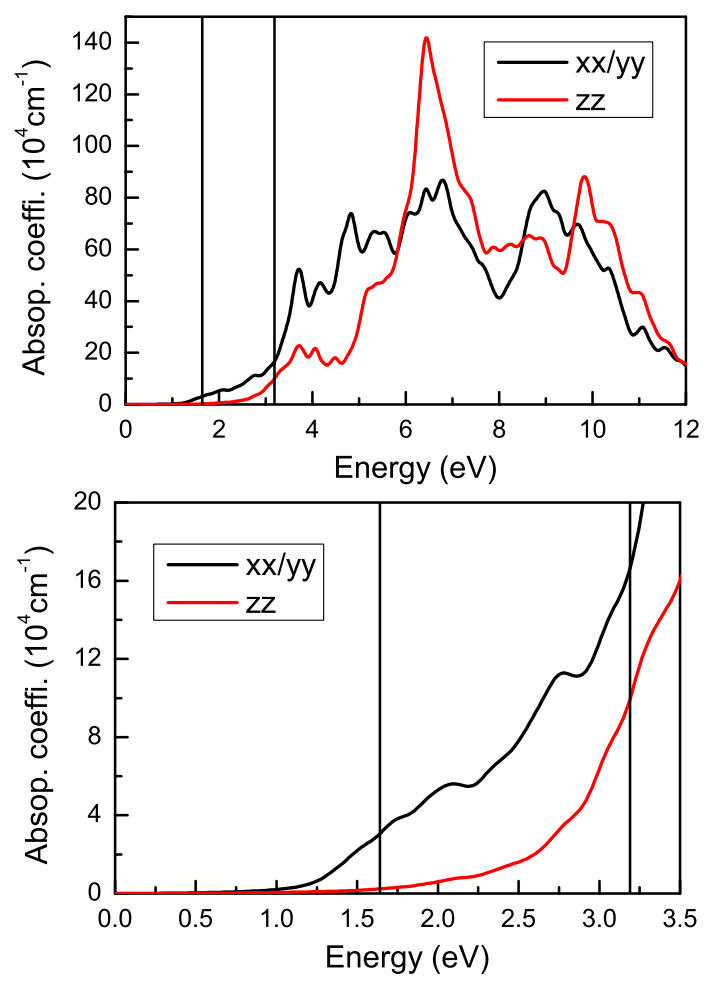

FIG. 8. (Color online)Using GGA+SOC, the optical absorption coefficients of SnSSe monolayer along xx/yy and $\mathrm{zz}$ directions from $0 \mathrm{eV}$ to $12 \mathrm{eV}$ (Top) and from $0 \mathrm{eV}$ to $3.5 \mathrm{eV}$ (Bottom), and the visible light region (1.6-3.1 eV) is shown.

Here, we use the rhombus primitive cell to investigate the optical properties of monolayer SnSSe. It is noted that the unit-cell volume $\Omega$ is not well-defined for $2 \mathrm{D}$ materials, and their optical properties depend on the the length of unit cell along $\mathrm{z}$ direction ${ }^{45}$. The dielectric function and absorption coefficient of SnSSe monolayer are calculated along $\mathrm{xx} / \mathrm{yy}$ and $\mathrm{zz}$ directions, and are plotted in Figure 7 and Figure 8. Along xx/yy and $\mathrm{zz}$ directions, strong anisotropy in the optical spectra can be observed due to distinct optical selection rules. In low frequency region, the $\varepsilon_{1}(\omega)$ along $\mathrm{xx} / \mathrm{yy}$ direction is higher than one along zz direction, The $\varepsilon_{2}(\omega)$ along $\mathrm{xx} / \mathrm{yy}$ direction shows a sharp increase at about $1.18 \mathrm{eV}$ at the first onset of the optical transitions, which is lower than one $(2.00 \mathrm{eV})$ along zz directin. The main peak region of $\varepsilon_{2}(\omega)$ along $\mathrm{xx} / \mathrm{yy}$ direction centres around $4 \mathrm{eV}$, and $6.3 \mathrm{eV}$ along $\mathrm{zz}$ direction. From the ultraviolet to the visible light region, strong absorption intensity can be observed along $\mathrm{xx} / \mathrm{yy}$ direction, and the absorption coefficient values reach up to more than $10^{4} \mathrm{~cm}^{-1}$ in the visible light region, implying high efficiency in the utilization of solar energy. The absorption intensity along $\mathrm{zz}$ direction is lower than one along $\mathrm{xx} / \mathrm{yy}$ direction in the visible light region. However, the very strong absorption intensity along zz direction is observed around $6.5 \mathrm{eV}$, which is higher than one along xx/yy direction. 


\section{ELECTRONIC TRANSPORT PROPERTIES}

Based on CSTA Boltzmann theory within rigid band approach (RBA), the electronic transport coefficients of SnSSe monolayer are calculated. To simulate the doping effects, the $\mathrm{n}(\mathrm{p})$-type doping is achieved by simply moving the position of Fermi level into conduction (valence) bands within RBA. Here, the rhombus primitive cell is used to investigate the electronic transport properties of SnSSe monolayer, and the studied transport direction is corresponding to zigzag (y) direction of the rectangle supercell. The Seebeck coefficient $\mathrm{S}$ is independent of relaxation time $\tau$, and the room temperature $\mathrm{S}$ as a function of doping level $(\mathrm{N})$ is shown in Figure 9 with both GGA and GGA+SOC. The SOC can reduce p-type S of SnSSe monolayer, and has little effect on $\mathrm{S}$ in n-type doping, which can be understood by considering SOC effects on the bands around the Fermi level. One reason is that the SOC can remove the valence band degeneracy near the VBM, leading to reduced p-type S. Another reason is that the SOC can reduce the effective masses of VBM (from Table II), producing a detrimental S in p-type doping due to:

$$
S=\frac{8 \pi^{2} K_{B}^{2}}{3 e h^{2}} m^{*} T\left(\frac{\pi}{3 n}\right)^{2 / 3}
$$

where $m^{*}, \mathrm{~T}$ and $n$ is the effective mass of the carrier, temperature and carrier concentration, respectively. Thus, it is very important for electronic transport studies to consider SOC. It is noted that the calculated electrical conductivity $\sigma / \tau$ and power factor $\mathrm{S}^{2} \sigma / \tau$ depend on $\tau$. By using calculated $\tau$ from Table II, the room temperature $\sigma$ and $\mathrm{S}^{2} \sigma$ as a function of $\mathrm{N}$ are shown in Figure 10, using GGA+SOC. For n-type doping, the $\tau=2.99 \times 10^{-14}$ $\mathrm{s}$, and $\tau=1.14 \times 10^{-13} \mathrm{~s}$ in p-type doping. Calculated results show that SnSSe monolayer has very excellent $\mathrm{S}^{2} \sigma$. It is found that the $\mathrm{n}$-type doping has better $\mathrm{S}^{2} \sigma$ than p-type one. Ultra low lattice thermal conductivity in monolayer $\mathrm{SnS}_{2}$ or $\mathrm{SnSe}_{2}$ has been predicted from a first principles study ${ }^{47}$. The lattice thermal conductivity of Janus MoSSe/PtSSe/ZrSSe monolayer is lower than $\mathrm{MoS}_{2} / \mathrm{PtS}_{2} / \mathrm{ZrS}_{2}$ monolayer ${ }^{29-31}$. Thus, the SnSSe monolayer should have ultra low lattice thermal conductivity, which suggests that Janus monolayer SnSSe may be a potential thermoelectric material.

\section{DISCUSSIONS AND CONCLUSION}

Monolayer $\mathrm{SnX}_{2}(\mathrm{X}=\mathrm{S}$, Se) have been recently synthesized $^{6,7}$, and Janus TMD monolayer MoSSe with sandwiched S-Mo-Se structure has also been synthesized by replacing the top $\mathrm{S}$ atomic layer in $\mathrm{MoS}_{2}$ with $\mathrm{Se}$ atoms $^{5,20}$. Thus, it is possible to achieve Janus SnSSe monolayer by replacing the top $\mathrm{S}(\mathrm{Se})$ atomic layer in $\mathrm{SnS}_{2}\left(\mathrm{SnSe}_{2}\right)$ with Se (S) atoms. In fact, growing Janus

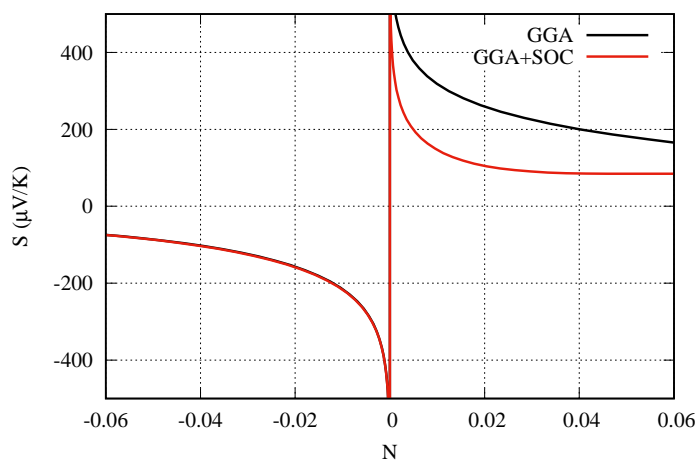

FIG. 9. (Color online) The room-temperature transport coefficient $\mathrm{S}$ of SnSSe monolayer as a function of doping level (N) using GGA and GGA+SOC.
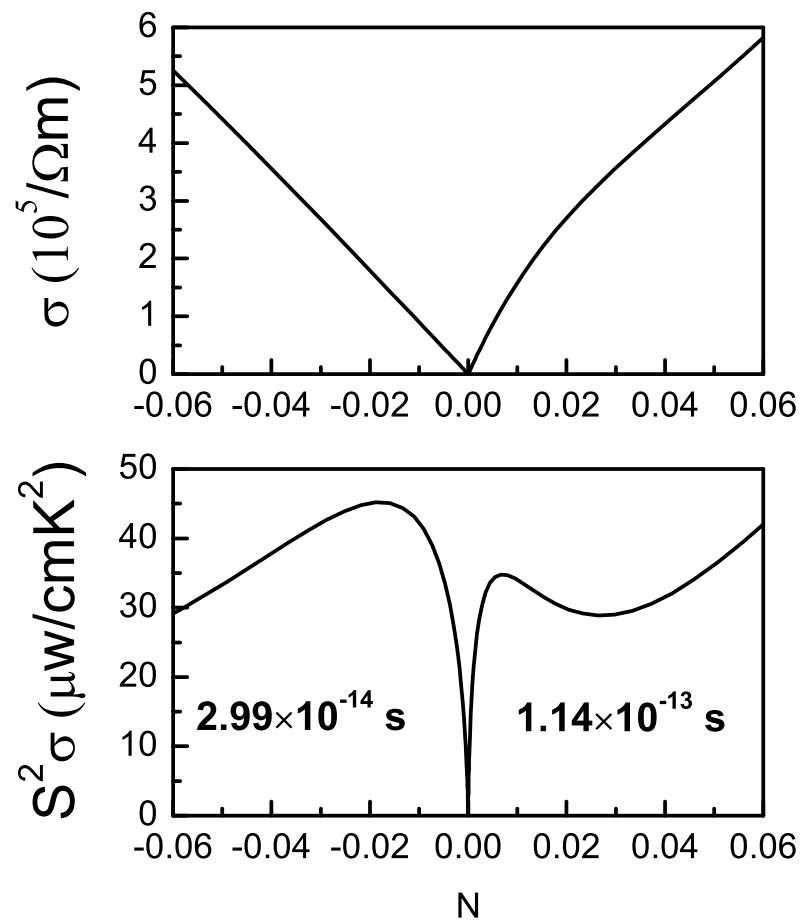

FIG. 10. The room-temperature transport coefficients $\sigma$ and $\mathrm{S}^{2} \sigma$ of SnSSe monolayer as a function of doping level $(\mathrm{N})$ using GGA+SOC.

SnSTe and SnSeTe monolayers is also possible. Compared to $\mathrm{SnS}_{2}$ and $\mathrm{SnSe}_{2}$ monolayers, the most important difference is that Janus $\mathrm{SnXY}(\mathrm{X} / \mathrm{Y}=\mathrm{S}, \mathrm{Se}, \mathrm{Te})$ monolayer breaks inversion symmetry, and therefore piezoelectricity can be produced. In addition to this, the electronic structures, phonons, elastic properties, optical properties and transport properties of $\mathrm{SnS}_{2}$ and $\mathrm{SnSe}_{2}$ monolayers can be tuned by constructing Janus monolayer. It has been proved that $\mathrm{SnS}_{2}$ and $\mathrm{SnSe}_{2}$ monolayers have ultra low lattice thermal conductivities, due to the low phonon velocity, low Debye temperature, weak bonding interactions and strong anharmonicity ${ }^{47}$. The Janus SnXY $(\mathrm{X} / \mathrm{Y}=\mathrm{S}, \mathrm{Se}, \mathrm{Te})$ monolayer should also have ultra low 
lattice thermal conductivity, and they may be promising candidates for thermoelectric applications.

In summary, the electronic structures, carrier mobilities, piezoelectric properties, optical properties and transport coefficients of Janus SnSSe monolayer are systematically studied from the reliable first-principle calculations. Janus monolayer SnSSe with $1 \mathrm{~T}$ phase is found to exhibit mechanical and dynamic stability and high experimental feasibility. Calculated results show that SnSSe monolayer is an indirect gap semiconductor, and the SOC can produce important influences on energy band structures, effective masses of VBM and electronic transport coefficients of Janus SnSSe monolayer. The hole mobilities of monolayer SnSSe are higher than electron ones, and are also higher than those of monolayer $\mathrm{SnS}_{2}$ or $\mathrm{SnSe}_{2}{ }^{47}$. The piezoelectric coefficient $d_{11}$ of SnSSe monolayer is comparable with one of $\alpha$-quartz commonly used 3D piezoelectric material ${ }^{52}$. The high absorption coefficients in the visible light region can be observed along xx/yy direction. The electronic transport calculations suggest that Janus monolayer SnSSe may be a potential thermoelectric material. Our works can stimulate further experimental works to synthesize SnSSe monolayer, and will motivate farther studies of other Janus monolayers, such as SnSTe and SnSeTe monolayers.

\section{ACKNOWLEDGMENTS}

This work is supported by the National Natural Science Foundation of China (Grant No. 11404391). We are grateful to the Advanced Analysis and Computation Center of China University of Mining and Technology (CUMT) for the award of CPU hours and WIEN2k/VASP software to accomplish this work.
${ }^{1}$ K. S. Novoselov et al., Science 306, 666 (2004).

2 J. P. Ji, X. F. Song, J. Z. Liu et al., Nat. Commun. 7, 13352 (2016).

3 S. Balendhran, S. Walia, H. Nili, S. Sriram and M.Bhaskaran, small 11, 640 (2015).

4 S. L. Zhang M. Q. Xie, F. Y. Li, Z. Yan, Y. F. Li, E. J. Kan, W. Liu, Z. F. Chen, H. B. Zeng, Angew. Chem. 128, 1698 (2016).

5 A. Y. Lu, H. Y. Zhu, J. Xiao et al., Nature Nanotechnology 12, 744 (2017).

6 X. Zhou, Q. Zhang, L. Gan, H. Li and T. Zhai, Adv. Funct. Mater. 26, 4405 (2016).

7 X. Zhou, L. Gan, W. M. Tian et al., Adv. Mater. 27, 8035 (2015).

8 M. Chhowalla, H. S. Shin, G. Eda, L. J. Li, K. P. Loh and H. Zhang, Nature Chemistry 5, 263 (2013).

${ }^{9}$ R. X. Fei, W. B. Li, J. Li and L. Yang, Appl. Phys. Lett. 107, 173104 (2015).

10 M. Bernardi, Ma. Palummo and J. C. Grossman, Nano Lett. 13, 3664 (2013).

11 W. X. Zhang, Z. S. Huang, W. L. Zhang and Y. R. Li, Nano Res. 7, 1731 (2014).

12 T. Cao, G. Wang, W. P. Han et al., Nat. Commun. 3, 887 (2012)

13 M. N. Blonsky, H. L. Zhuang, A. K. Singh and R. G. Hennig, ACS Nano, 9, 9885 (2015).

14 W. Huang, H. X. Da and G. C. Liang, J. Appl. Phys. 113, 104304 (2013).

15 G. P. Li, G. Q. Ding and G. Y. Gao, J. Phys.: Condens. Matter 29, 015001 (2017).

16 S. D. Guo and J. L. Wang, Semicond. Sci. Tech. 31, 095011 (2016).

17 H. Y. Lv, W. J. Lu, D. F. Shao, H. Y. Lub and Y. P. Sun, J. Mater. Chem. C 4, 4538 (2016).

18 S. D. Guo, J. Mater. Chem. C 4, 9366 (2016).

19 A. Shafique and Y. H. Shin, Phys. Chem. Chem. Phys. 19, 32072 (2017).

20 J. Zhang, S. Jia, I. Kholmanov et al., ACS Nano 118, 8192 (2017).
${ }^{21}$ L. Dong, J. Lou and V. B. Shenoy, ACS Nano 11, 8242 (2017).

22 M. Yagmurcukardes, C. Sevik and F. M. Peeters, Phys. Rev. B 100, 045415 (2019).

${ }^{23}$ W. J. Yin, B. Wen, G. Z. Nie and X. L. Wei and L. M. Liu, J. Mater. Chem. C 61693 (2018).

24 W. W. Shi and Z. G. Wang, J. Phys.: Condens. Matter 30, 215301 (2018).

25 Y. C. Cheng, Z. Y. Zhu, M. Tahir et al., Europhys. Lett. 102, 57001 (2013).

${ }^{26}$ X. C. Ma, X. Wu, H. D. Wang and Y. C. Wang, J. Mater. Chem. A 6, 2295 (2018).

27 R. Peng, Y. D. Ma, B. B. Huang and Y. Dai, J. Mater. Chem. A 7, 603 (2019).

28 F. P. Li, W. Wei, P. Zhao, B. B. Huang and Y. Dai, J. Phys. Chem. Lett. 8, 5959 (2017).

29 S. D. Guo, Phys. Chem. Chem. Phys. 20, 7236 (2018).

30 S. D. Guo, Y. F. Li and X. S. Guo, Comp. Mater. Sci. 161, 16 (2019).

31 W. L. Tao, Y. Mu, C. E. Hu, Y. Cheng and G. F. Ji, Philosophical Magazine, 99, 1025 (2019).

32 P. Hohenberg and W. Kohn, Phys. Rev. 136, B864 (1964); W. Kohn and L. J. Sham, Phys. Rev. 140, A1133 (1965).

33 P. Blaha, K. Schwarz, G. K. H. Madsen, D. Kvasnicka and J. Luitz, WIEN2k, an Augmented Plane Wave + Local Orbitals Program for Calculating Crystal Properties (Karlheinz Schwarz Technische Universität Wien, Austria) 2001, ISBN 3-9501031-1-2

34 J. P. Perdew, K. Burke and M. Ernzerhof, Phys. Rev. Lett. 77, 3865 (1996).

35 A. H. MacDonald, W. E. Pickett and D. D. Koelling, J. Phys. C 13, 2675 (1980).

36 D. J. Singh and L. Nordstrom, Plane Waves, Pseudopotentials and the LAPW Method, 2nd Edition (Springer, New York, 2006).

37 J. Kunes, P. Novak, R. Schmid, P. Blaha and K. Schwarz, Phys. Rev. B 64, 153102 (2001).

38 D. D. Koelling, B. N. Harmon, J. Phys. C: Solid State Phys. 10, 3107 (1977). 
39 G. K. H. Madsen and D. J. Singh, Comput. Phys. Commun. 175, 67 (2006).

40 G. Kresse, J. Non-Cryst. Solids 193, 222 (1995).

41 G. Kresse and J. Furthmüller, Comput. Mater. Sci. 6, 15 (1996).

42 G. Kresse and D. Joubert, Phys. Rev. B 59, 1758 (1999).

43 A. Togo, F. Oba, and I. Tanaka, Phys. Rev. B 78, 134106 (2008).

44 X. Wu, D. Vanderbilt and D. R. Hamann, Phys. Rev. B 72, 035105 (2005).

${ }^{45}$ G. Y. Guo, K. C. Chu, D. S. Wang and C. G. Duan, Phys. Rev. B 69, 205416 (2004).

46 X. F. Wu, V. Varshney et al., Chem. Phys. Lett. 669, 233 (2017).

47 A. Shafique, A. Samad and Y. H. Shin, Phys. Chem. Chem.
Phys. 19, 20677 (2017).

48 R. C. Andrew, R. E. Mapasha, A. M. Ukpong and N. Chetty, Phys. Rev. B 85, 125428 (2012).

49 E. Cadelano, P. L. Palla, S. Giordano and L. Colombo, Phys. Rev. B 82, 235414 (2010).

50 S. D. Guo and J. Dong, Semicond. Sci. Tech. 33, 085003 (2018).

51 S. Bruzzone and G. Fiori, Appl. Phys. Lett. 99, 222108 (2011).

52 R. Bechmann, Phys. Rev. 110, 1060 (1958).

53 M. Gajdos, K. Hummer, G. Kresse, J. Furthmuller and F. Bechstedt, Phys. Rev. B 73, 045112 (2006).

54 X. Huang, T. R. Paudel, S. Dong and E. Y. Tsymbal, Phys. Rev. B 92, 125201 (2015). 